\title{
Bone morphogenetic protein 4 (BMP4) loss-of-function variant associated with autosomal dominant Stickler syndrome and renal dysplasia
}

\author{
Thomas R. W. Nixon $\mathbb{1}^{1,2} \cdot$ Allan Richards ${ }^{3} \cdot$ Laura K. Towns $^{3} \cdot$ Gavin Fuller $^{4} \cdot$ Stephen Abbs $^{4} \cdot$ Philip Alexander $^{2}$. \\ Annie McNinch $^{3} \cdot$ Richard N. Sandford ${ }^{4} \cdot$ Martin P. Snead $^{1,2}$
}

Received: 5 April 2018 / Revised: 5 November 2018 / Accepted: 27 November 2018 / Published online: 19 December 2018

(c) The Author(s) 2018. This article is published with open access

\begin{abstract}
Stickler syndrome is a genetic disorder that can lead to joint problems, hearing difficulties and retinal detachment. Genes encoding collagen types II, IX and XI are usually responsible, but some families have no causal variant identified. We investigate a variant in the gene encoding growth factor BMP4 in a family with Stickler syndrome with associated renal dysplasia. Next generation sequencing of the coding region of COL2A1, COL11A1 and a panel of genes associated with congenital anomalies of the kidney and urinary tract (CAKUT) was performed. A novel heterozygous BMP4 variant causing a premature stop codon, c. 130G $>$ T, p.(Gly44Ter), which segregated with clinical features of Stickler syndrome in multiple family members, was identified. No variant affecting gene function was detected in COL2A1 or COL11A1. Skin fibroblasts were cultured with and without emetine, and the mRNA extracted and analysed by Sanger sequencing to assess whether the change was causing nonsense-mediated decay. Nonsense-mediated decay was not observed from the extracted BMP4 mRNA. BMP4 is a growth factor known to contribute to eye development in animals, and gene variants in humans have been linked to microphthalmia/anophthalmia as well as CAKUT. The variant identified here further demonstrates the importance of BMP4 in eye development. This is the first report of a BMP4 DNA variant causing Stickler syndrome, and we suggest $B M P 4$ be added to standard diagnostic gene panels for this condition.
\end{abstract}

\section{Introduction}

Stickler syndrome is a genetic disorder affecting the eyes, joints, hearing and orofacial development. It is associated

Supplementary information The online version of this article (https:// doi.org/10.1038/s41431-018-0316-y) contains supplementary material, which is available to authorized users.

$\triangle$ Martin P. Snead

mps34@cam.ac.uk

1 School of Clinical Medicine, University of Cambridge, Addenbrooke's Hospital, Hills Road, Cambridge CB2 0SP, UK

2 Vitreoretinal Service, Addenbrooke's Hospital, Hills Road, Cambridge University Hospitals NHS Foundation Trust, Cambridge CB2 0QQ, UK

3 Department of Pathology, University of Cambridge, Tennis Court Road, Cambridge CB2 1QP, UK

4 East Anglian Medical Genetics Service, Box 134, Addenbrooke's Treatment Centre, Hills Road, Cambridge CB2 0QQ, UK with a significantly increased lifetime risk of rhegmatogenous retinal detachment and is the most common cause of non-traumatic retinal detachment in children. Other ocular features include myopia, often severe, and congenital defects of the vitreous, which can be a useful diagnostic feature and helps direct genetic testing $[1,2]$. Hearing loss, present in $63 \%$ of patients, is usually high frequency sensorineural loss, with a variable conductive component in children due to intermittent otitis media [3]. Joint problems, in $75 \%$, include hypermobility and premature osteoarthritis leading to chronic back pain and early joint replacement surgery [4]. Orofacial manifestations include cleft palate (41\%), Pierre Robin sequence (24\%) and midfacial hypoplasia [5, 6]. There is, however, marked variability in the degree to which each system is affected in different patients [6].

Most cases of Stickler syndrome are due to variants in genes encoding various fibrillar collagens. Type 1 Stickler syndrome is the most common, accounting for $\sim 80 \%$ of the cases, and is due to heterozygous changes in COL2AI (ref. 2), which encodes the alpha-1 chain of type II 
collagen. It carries the greatest risk of retinal detachment, but generally milder hearing loss. Type 2 Stickler syndrome is caused by heterozygous alteration to COL11Al (ref. 7), which encodes the alpha-1 chain of type XI collagen. Type 3 Stickler syndrome is caused by heterozygous changes to COL11A2 (ref. 8), which is not expressed in the eye, resulting in a non-ocular phenotype. There is an ocular-only variant caused by variants in COL2A1 affecting exon 2 (ref. 9), which is only expressed in the eye. Less frequently, recessive Stickler syndrome has been found to be caused by homozygous variants in COL9A1 (ref. 10), COL9A2 (ref. 11) and COL9A3 (ref. 12), and bi-allelic COL11A1 sequence changes [13].

Stickler syndrome has been associated with recessive variants in two non-collagen genes: one family with a homozygous variant in LOXL3 (ref. 14), which encodes an enzyme involved in collagen crosslinking and one family with a homozygous variant in $L R P 2$ (ref. 15), encoding an endocytic transmembrane receptor.

The bone morphogenetic proteins (BMPs) are growth factors that are part of the TGF- $\beta$ superfamily [16]. They are expressed throughout embryogenesis and have diverse roles in development. Initially identified and named for their role in osteogenesis, they are now recognised to have a crucial role in early embryonic development in gastrulation and neurulation, mesoderm patterning, and cell proliferation, differentiation and morphogenesis in many organ systems. BMP4 is known to have a critical role in ocular development in establishing the dorsal-ventral axis of the developing eye [17] as well as lens induction and retinal development [18]. Humans with loss-of-function variants in $B M P 4$ have previously been described with highly variable manifestations, including anophthalmia/ microphthalmia, syndactyly and structural brain anomalies including thinning of the corpus callosum and widened cerebral sulci $[19,20]$. Deletions of the $14 \mathrm{q} 22-$ q23 region, which includes BMP4 as well as $O T X 2$, also known to be important for eye development, have been described causing anophthalmia/microphthalmia and also hearing problems, Pierre-Robin sequence, developmental delay and cleft palate [21-23]. Mice models with bmp4 loss-of-function variants have features including microphthalmia, craniofacial anomalies, polydactyly and congenital anomalies of the kidney and urinary tract (CAKUT) [24].

In this study, we investigate a family with clinical features of Stickler syndrome, but in which one member of the family also had congenital renal dysplasia leading to renal replacement therapy at a young age. Next generation sequencing identified a sequence variant in the gene for $B M P 4$, which segregated with the Stickler syndrome phenotype in this family.

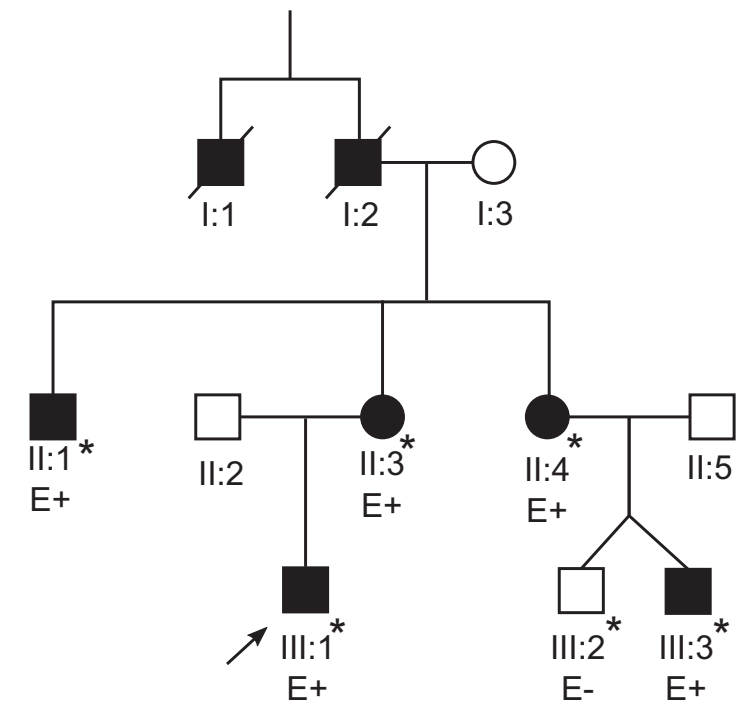

Fig. 1 Family pedigree. Patients clinically affected are in solid. * Ophthalmologically evaluated clinically (Martin Snead and Thomas Nixon). E+ DNA evaluated as positive for familial BMP4 variant. EDNA evaluated as negative for familial BMP4 variant

\section{Patient data}

One male, aged 20, with renal dysplasia requiring renal replacement therapy was referred to clinical genetics (Patient III:1 in Fig. 1, Table 1). It was noted that several family members had a clinical diagnosis of Stickler syndrome, with five affected members over two generations (Patients I:1, I:2, II:1, II:3, II:4 in Fig 1, Table 1). Previous sequencing of the COL2A1 and COL11A1 genes in one of the affected family members had not identified a variant that would affect function. The clinical features present in the family included high myopia, congenital hypoplasia of the vitreous, retinal detachment, sensorineural hearing loss (Fig. 2), retrognathia and high-arched palate (Fig. 3). As a result, this patient was referred to the National Stickler syndrome diagnostic service, which confirmed that he also had myopia with abnormal vitreous architecture. Two further family members were examined (III:2 and III:3), of which one had myopia with abnormal vitreous and the other had normal ophthalmological examination. No other family members reported kidney disease. Renal ultrasound was offered but was declined.

\section{Methods}

\section{Genetic analysis}

Next generation sequencing was performed using the TruSight One sequencing kit (Illumina, Cambridge, United Kingdom). Variants in a panel of genes known to cause 


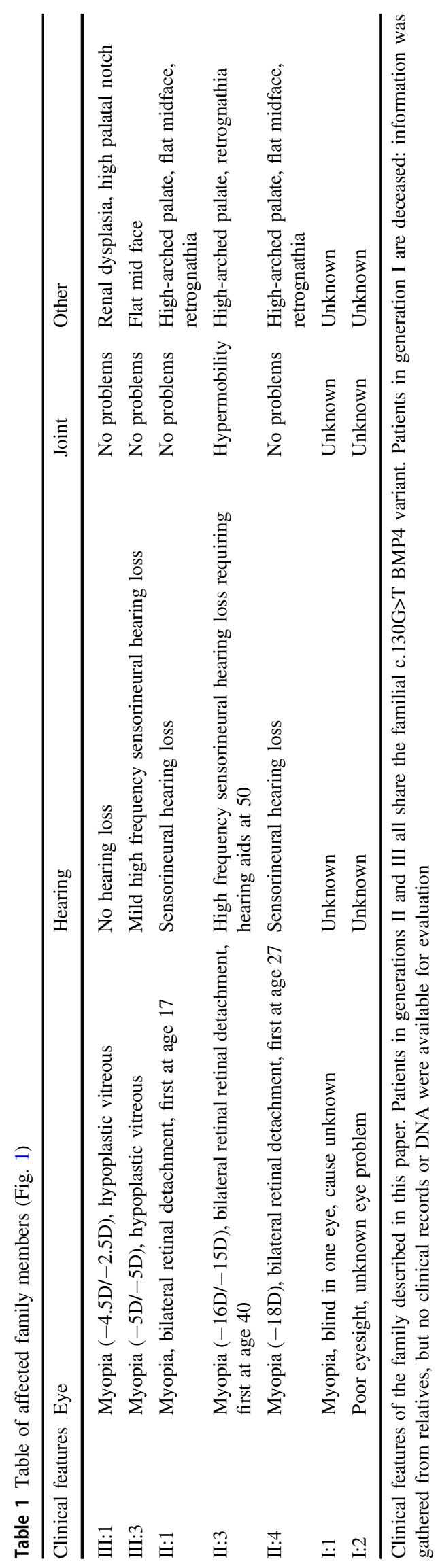

CAKUT (see supplementary information) were identified. 99.69\% of the target sequence within this panel was sequenced to a depth of 20 -fold or more, with analytical sensitivity of $98.3 \%-100 \%$ (95\% Confidence Intervals). Further analysis identified a heterozygous loss-of-function variant in the BMP4 (NM_001202.3) gene: c.130G $>$ T, p. (Gly44Ter). DNA variant analysis conformed to best practice guidelines [25]. Sanger sequencing was used to confirm the sequence change and to analyse additional family members. These variants were submitted to the LOVD database and can be found at www.LOVD.nl/BMP4 (patient IDs 155750, 165221, 165222, 165224 and 165225).

\section{mRNA analysis}

RNA was extracted using RNeasy column, (Qiagen, Manchester, United Kingdom) from cultured dermal fibroblasts (family member II-3), that had been incubated either with or without emetine, an inhibitor of nonsense-mediated decay (as previously described) [26]. Reverse-transcription PCR was performed using Superscript II Reverse Transcriptase (Invitrogen, Hemel Hempstead, United Kingdom) and an antisense primer: 5'-TGTAGTGTGTGGGTGAGTG GATGG-3'. PCR amplification was then performed of a 940 bp region of the BMP4 cDNA surrounding the variant, using Q5 High-fidelity DNA polymerase (NEB, Hitchin, United Kingdom) in reaction conditions as recommended by the manufacturer. A primary reaction used primers $5^{\prime}$ ATGATTCCTGGTAACCGAATGCTG- $3^{\prime}$ in exon 3, and $5^{\prime}$-ACCTTATCATACTCATCCAGGTAC-3' in exon 4. Products from this primary reaction were then re-amplified in a secondary reaction using primers 5'-ATTATGC CAAGTCCTGCTAGGAGG- $3^{\prime}$ in exon 3 , and $5^{\prime}$ GGGCCACAATCCAGTCATTCCAGC- $3^{\prime}$ in exon 4. The resulting cDNA product was sequenced using the primer $5^{\prime}$ ATGTTCTTCGTGGTGGAAGCTC-3' using standard methods for Sanger sequencing.

\section{Results}

\section{Sequencing}

Next generation sequencing of the patient identified a heterozygous sequence change in the BMP4 gene: NM_001202.3: c.130G $>$ T, p.(Gly44Ter). This is predicted to cause a premature termination codon. Sanger sequencing confirmed that this variant was present in family members with the Stickler syndrome phenotype and not present in those with a normal phenotype (Fig. 4). The sequence change was not present in public DNA sequence variation databases (http://exac.broadinstitute.org/; http://www. internationalgenome.org/). 


\section{II:1 Audiogram (age 41)}

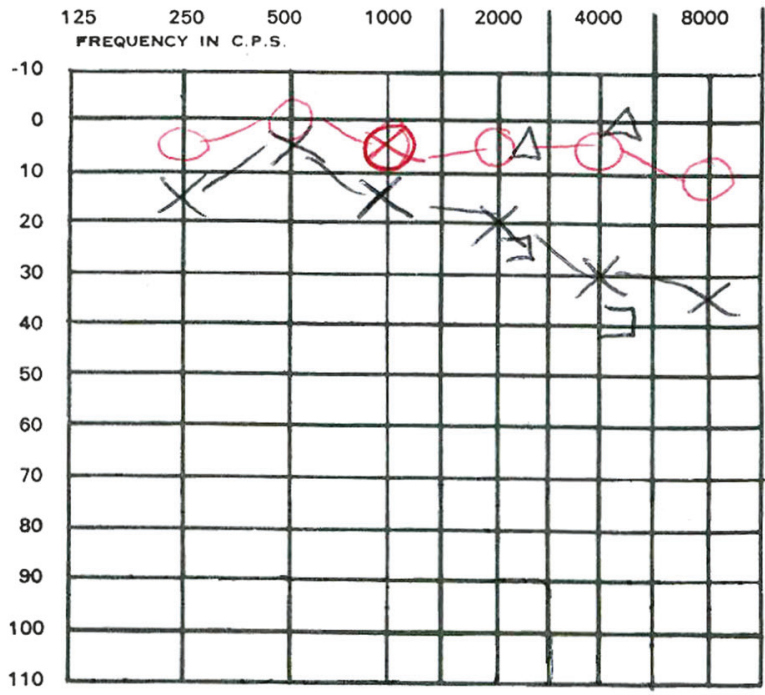

II:4 Audiogram (age 38)

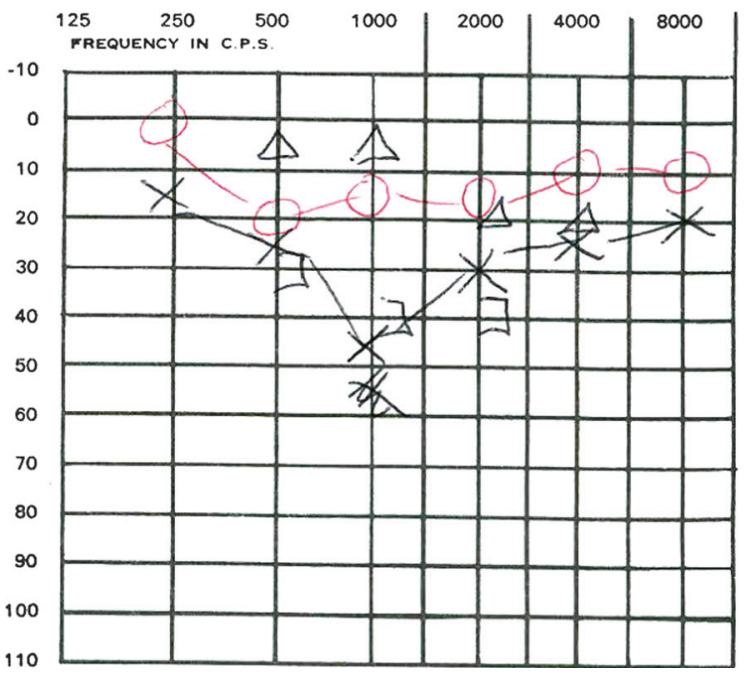

II:3 Audiogram (age 40)

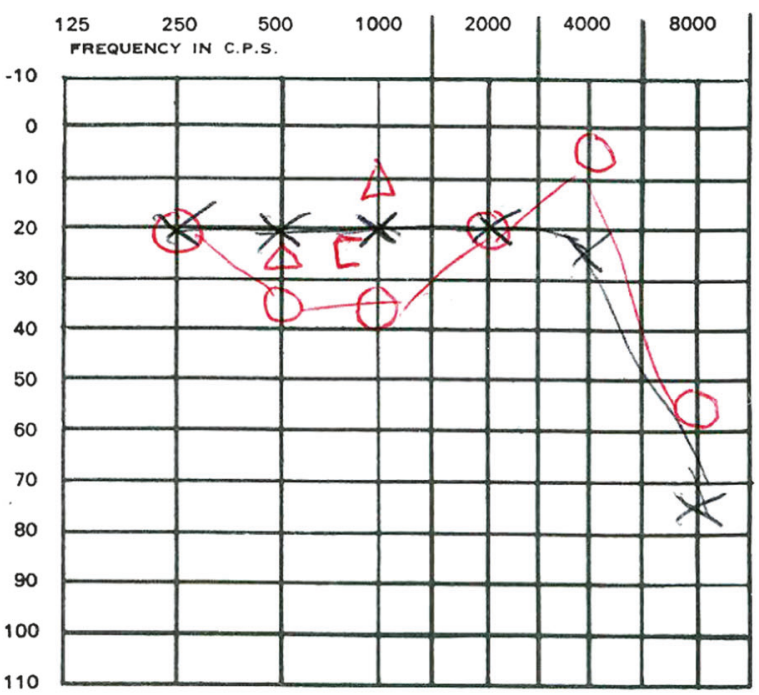

III:3 Audiogram (age 19)

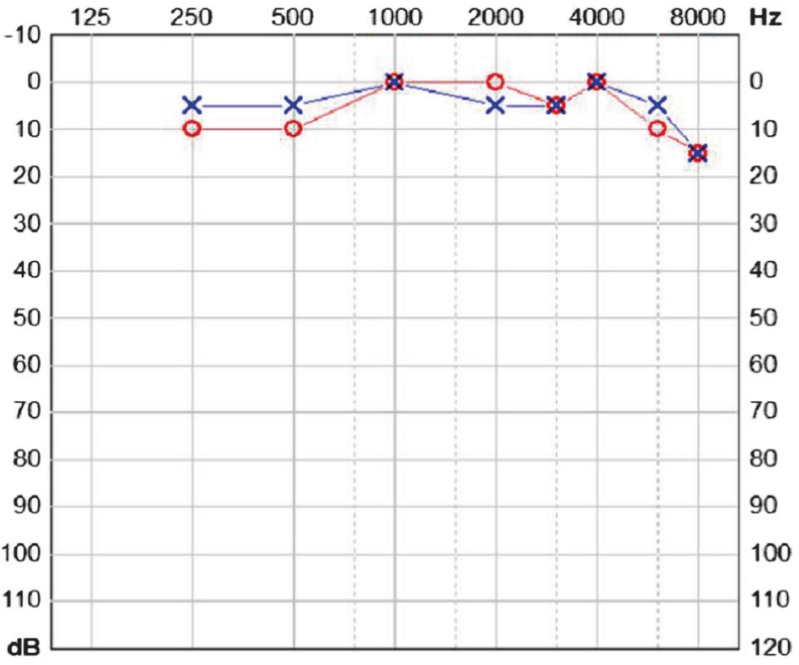

Symbols:

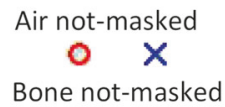

$\triangle \Delta$

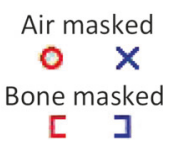

[ ]

Fig. 2 Audiograms. Pure tone audiometry thresholds for patients II:1, II:3, II:4 and III:3

\section{mRNA analysis}

Sanger sequencing of the RT-PCR products demonstrated that both variant and wild-type sequences were present (Fig. 5) demonstrating that the variant did not result in nonsense-mediated decay, but would result in a severely truncated protein.

\section{Discussion}

We have described a novel variant in a gene, BMP4, associated with a Stickler syndrome phenotype. The vast majority of patients with dominant Stickler syndrome are found to have COL2A1 or COL11A1 variants [2], and in our experience less than $5 \%$ of patients that have a clear clinical 


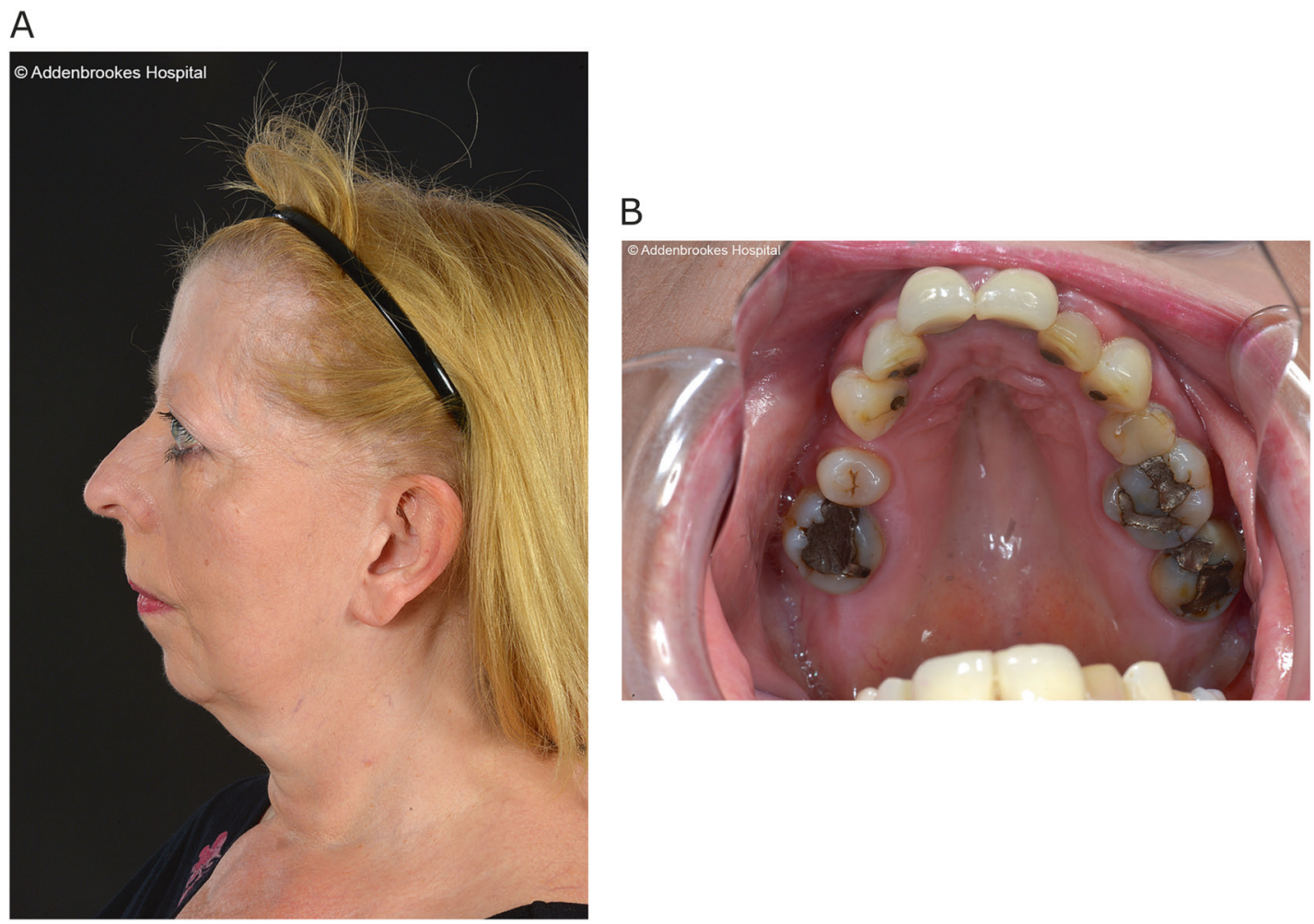

Fig. 3 Clinical photographs of patient II:3. a Right lateral facial photograph of patient II:3 demonstrating retrognathia. b Intra-oral photograph of patient II:3 demonstrating high-arched palate

diagnosis have no detectable variants that affect function in either of these two genes, or those encoding type IX collagen. This is the first description of a BMP4 gene variant associated with a clinical phenotype of Stickler syndrome and is the first non-collagen sequence change found to be associated with dominant Stickler syndrome, although recessive Stickler or Stickler-like syndromes have been described in two other non-collagen genes, LOXL3 (ref. 14) and $L R P 2$ (ref. 15). One consanguineous family with two children diagnosed with Stickler syndrome, cleft palate, micrognathia and congenital high myopia were found to have a homozygous change in LOXL3, which encodes the enzyme lysyl oxidase-like 3. Lysyl oxidase-like 3 is known to cross-link type II collagen, which underlines the fundamental collagenopathy in Stickler syndrome. A further consanguineous family with two children diagnosed with Stickler syndrome, with congenital high myopia, hypoplastic vitreous, a possible vitreous membranous anomaly similar to type 1 Stickler syndrome, giant retinal tear detachment and joint hypermobility, were found to have a homozygous change in $L R P 2$. LRP2 encodes lipoprotein receptor-related protein-2, an endocytic transmembrane receptor. Variants in LRP2 are known to cause DonnaiBarrow syndrome, a rare autosomal recessive disorder featuring agenesis of the corpus callosum, sensorineural hearing loss, facial dysmorphism and ocular findings, including myopia and coloboma. The pathway to cause Stickler syndrome is less clear in this gene, but it likely interferes with intercellular signalling during embryogenesis.

Like other members of the TGF- $\beta$ superfamily, BMP4 is synthesised as a precursor molecule and is proteolytically cleaved into two domains, an $\mathrm{N}$-terminal prodomain, (which can vary in length due to different transcripts) and a C-terminal active growth factor dimer forming region [27]. As well as homodimers, BMP4 also forms heterodimers with BMP7, with the heterodimers being more active [28]. Heterozygous BMP7 loss-of-function variants have also been associated with anophthalmia [29].

With a 24 amino acid signal peptide, the c. $130 \mathrm{G}>\mathrm{T}$, p. (Gly44Ter) [transcript variant NM_001347912.1; c. $271 \mathrm{G}>\mathrm{T}$, p.(Gly91Ter)] variant we describe here results in only 19 (or 66 from the longer transcript, Fig. 6) amino acids of the BMP4 prodomain being expressed (the mRNA does not undergo nonsense-mediated decay). This may explain why, when compared with previously described $B M P 4$ variants, the ocular phenotype in this family is mild. The majority of documented BMP4 loss-of-function variants have severe ocular anomalies, including anophthalmia, microphthalmia, and anterior chamber abnormalities with congenital glaucoma [19-23]. Systemic features are variable and include developmental delay, brain and pituitary 


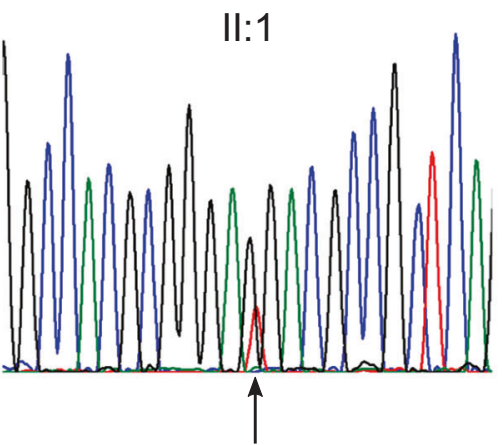

III:1

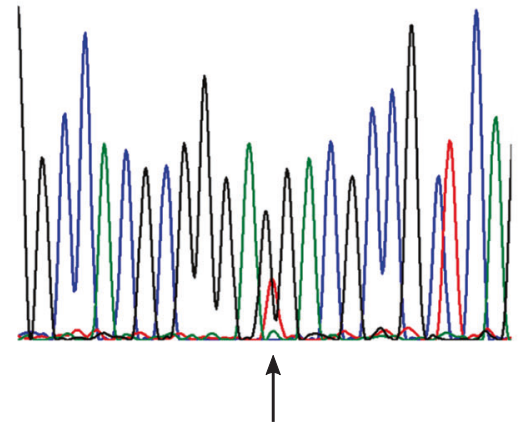

II:3

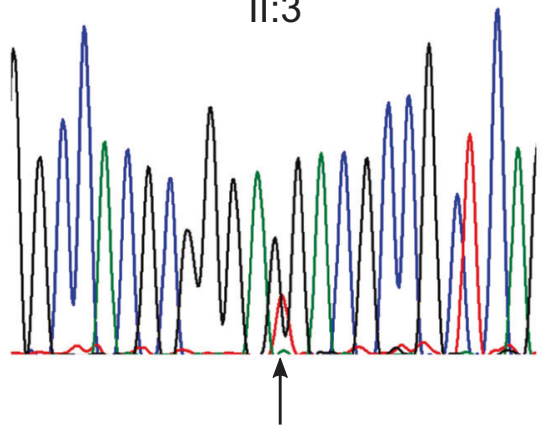

III:2

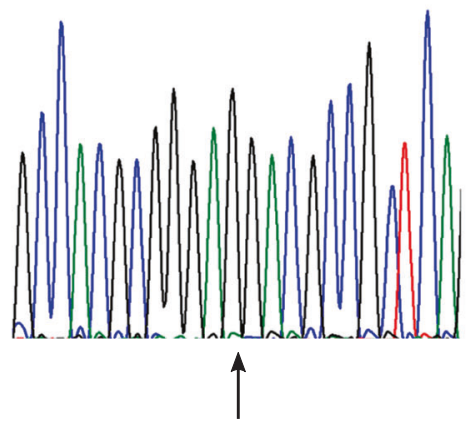

II:4

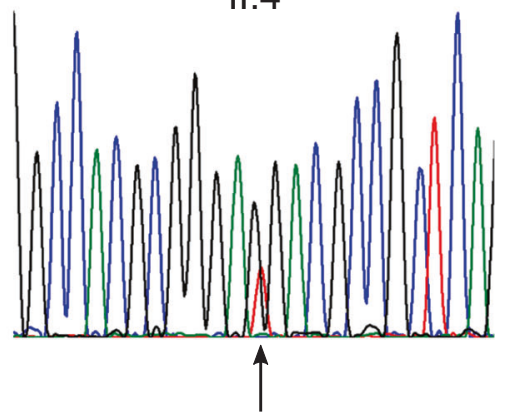

III:3

Fig. 4 Sequencing chromatographs for tested family members. Chromatographs demonstrating a heterozygous G>T BMP4 substitution (arrowed) in affected family members, with no substitution (arrowed) in unaffected family member (III:2)

A
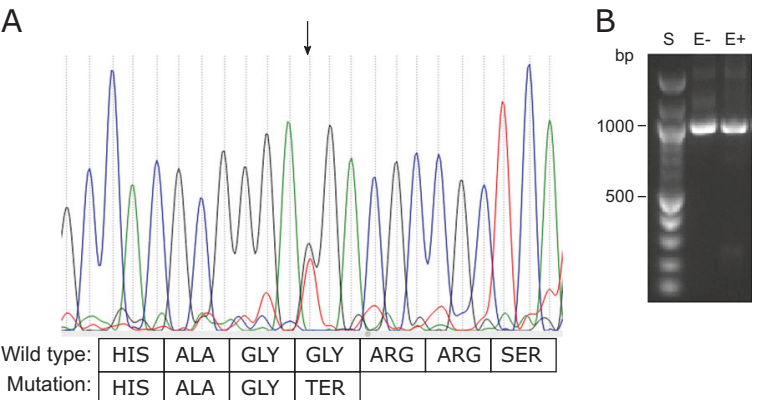

Fig. 5 Sequencing chromatograph from RT-PCR. a Sanger sequencing of RT-PCR products (sense orientation) demonstrating heterozygosity in mRNA extracted from untreated (- emetine) cultured dermal fibroblasts at the variant locus, i.e. nonsense-mediated decay does not take place. The $\mathrm{G}>\mathrm{T}$ variant (arrowed) changes a GGA coding a glycine residue into a premature stop codon. b Agarose gel of PCR products. S size ladder. E- sample from cells cultured without emetine, $\mathrm{E}+$ sample from cells cultured with emetine. PCR products are of expected cDNA size of $\sim 1 \mathrm{~kb}$

anomalies, craniofacial dysmorphism, kidney anomalies and polydactyly. Those previously described variants associated with disease were downstream of the variant in the pedigree described here. It is possible, therefore, that those changes produced an abnormal protein with a dominant negative effect, not present in this pedigree, which in this case may simply reflect haploinsufficiency of BMP4 (due to lack of functional protein from severe truncation).
Interestingly the c.171dupC, p.(Glu58Argfs*17) [NM_001347912.1; c.312dupC, p.(Glu105Argfs*17)] $B M P 4$ variant only encodes an additional 14 normal amino acids of the prodomain yet that change results in a severe ocular phenotype [20]. BMP4 prodomains can form a stable interaction with the active domains and alter their affinity with extracellular matrix molecules [28]. The difference in resulting phenotypes between c. $130 \mathrm{G}>\mathrm{T}$, p.(Gly44Ter) and c.171dupC, p.(Glu58Argfs*17) may therefore reflect the ability of these truncated prodomains to form a complex with growth factor dimers and produce a dominant-negative effect. It is interesting to contrast the microphthalmia/ anophthalmia seen in previously described BMP4 variants with the megalophthalmos and myopia seen in this pedigree.

Another mechanism that potentially modifies the phenotype, which needs to be considered is that not all BMP4 transcripts contain the variant described here (Fig. 6). Transcripts NM_001347913.1, NM_001347915.1, NM_001347917.1, all have the initiating methionine downstream of the c.130G $>\mathrm{T}$ variant and do not encode the first 63 amino acids of the NM_01202.3 transcript. However, this is also true for the c.171dupC p.(Glu58Argfs*17) variant and so is unlikely to be a major consideration.

A number of cases involve microdeletions, including BMP4 and adjacent genes in the $14 \mathrm{q} 22-23$ region. OTX2 is only $2.8 \mathrm{Mbs}$ from $B M P 4$ and is also critical in eye 


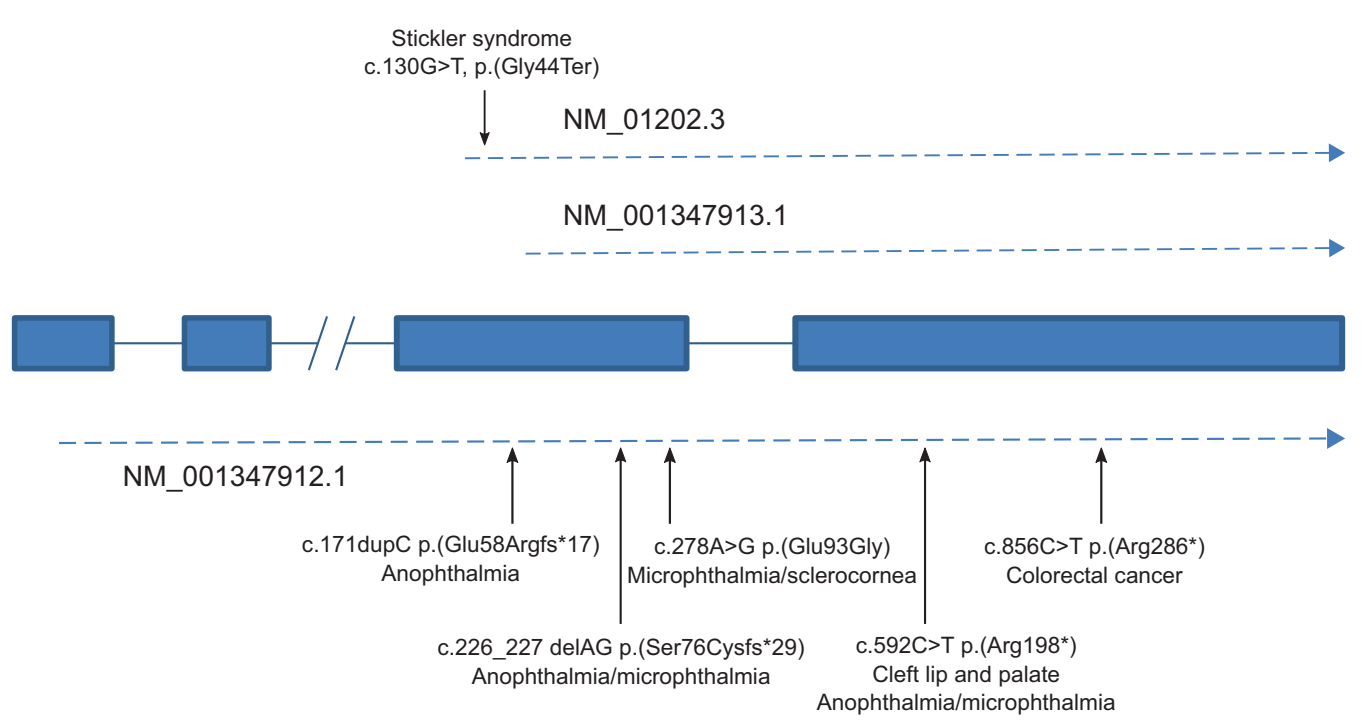

Fig. 6 BMP4 transcripts and variant positions. The BMP4 gene consists of four exons. Transcription can start at multiple locations on the gene. All variants are described relative to their position on transcript NM_01202.3. Three different BMP4 transcripts are shown, demonstrating the position of the $c .130 \mathrm{G}>\mathrm{T}$ variant compared with those

development, with variants also known to cause microphthalmia/anophthalmia. Concurrent deletions of both genes have been associated with microphthalmia/anophthalmia with decreased cerebral white matter volume [30].

BMP4 has been shown to be critical in establishing the dorsal-ventral axis of the developing eye [17]. In the chick eye, bmp4 is expressed in the dorsal optic cup and is responsible for spatiotemporal co-ordination of apoptotic cell death [31]. In Xenopus, bmp4 has been shown to act via inducing tbx 5 to determine the dorsal region of the optic cup, with the factor sonic hedgehog having an opposing effect [17]. In mouse embryos, increased levels of bmp4 were shown to reduce retinal volume, alter the shape of the optic cup and reduce axial length - this suggests that low levels of BMP4 could contribute to increased axial length and therefore congenital myopia [32]. BMP4 is also critical for lens induction [19].

The interaction between BMP4 and TGF $\beta 1$ may be important in its role in pathogenesis of retinal detachment. It is known that exon 2 of COL2A1, expressed in the eye and developing cartilage but not mature cartilage, encodes a region of type II procollagen with a cysteine-rich domain that binds TGF $\beta 1$ and BMP2 (ref. 33). Loss-of-function variants in COL2Al cause type 1 Stickler syndrome, with vitreous anomaly and significant risk of retinal detachment. Additionally, intronic sequence variants affecting the alternative splicing efficiency of exon 2 of COL2Al have been associated with an increased risk of retinal detachment [34]. BMP4 and BMP2 have significant structural similarity so it is possible that BMP4 can also bind to type II collagen previously described. Transcript NM_001347913.1 is unaffected by this variant. All previously described variants are downstream of the variant described in this paper and all affect the NM_001347913.1 transcript, except c.171dupC

[35]. TGF $\beta 1$ has also been shown to bind to type IV collagen [36]. This is an important structural component of the inner limiting membrane of the retina, which then contributes to the posterior hyaloid membrane of the vitreous [37], which if too adherent to the retina can cause a retinal tear leading to retinal detachment. Fibrillin is a major component of microfibrils in extracellular matrices, and variants in the fibrillin gene $(F B N I)$ can cause Marfan syndrome, which has many features including myopia and risk of retinal detachment. It has been shown that mice deficient in fibrillin have dysregulation of TGF $\beta 1$ (ref. 38). It has also been demonstrated that fibrillin assemblies have a role in directing signalling through TGF $\beta 1$ and BMP pathways [39]. This may suggest that TGF $\beta 1$ dysregulation may be a 'final common pathway' in the pathogenesis of retinal detachment in patients with a genetic predisposition.

The c.130G $>$ T BMP4 variant was initially identified in the proband through testing a panel of genes known to be associated with CAKUT. No other family members are known to have renal disease but detailed imaging has not been carried out in all individuals who have the BMP4 variant. The aetiology of CAKUT is complex. A family history of CAKUT is found in about 10 to $20 \%$ of cases but only a minority will have an identifiable genetic cause identified. Where a genetic cause is identified, the pattern of inheritance and segregation of the disease phenotype typically appears as an autosomal dominant trait with incomplete penetrance and variable expression. This marked variability, also seen in animal models of CAKUT, may be due to a number of different factors including hypomorphic 
variants that do not completely disrupt early nephrogenesis but affect renal and urinary tract development at later stages of gestation and genetic or environmental modifiers [40].

In summary, a BMP4 variant has been identified as a cause of autosomal dominant Stickler syndrome. Molecular genetic diagnosis is important for families to allow appropriate follow-up, risk management advice and genetic counselling regarding family planning, so testing for BMP4 should be considered in Stickler syndrome where no variant is identified in a collagen gene that would be expected to affect function. If a loss-of-function BMP4 variant is identified in a patient with Stickler syndrome then patients should be investigated for CAKUT, and conversely if identified in a patient with CAKUT, they should be referred for ophthalmological assessment.

Acknowledgements We thank the family for participating. We thank the staff at the NHS Medical Genetics laboratory at Addenbrooke's Hospital for technical and interpretative assistance. We thank Adrian Blackwell and Senjah Brown, clinic co-ordinators to the National Stickler syndrome diagnostic service, for administrative support. Thomas R W Nixon and the Vitreoretinal Research Group are supported by the Retinal Research Fund at the University of Cambridge. The University of Cambridge has received salary support in respect of Richard N Sandford from the NHS in the East of England through the Clinical Academic Reserve. The views expressed are those of the authors and not necessarily those of the NHS or Department of Health.

Funding Thomas R W Nixon and the Vitreoretinal Research Group are supported by the Retinal Research Fund at the University of Cambridge. The University of Cambridge has received salary support in respect of Richard N Sandford from the NHS in the East of England through the Clinical Academic Reserve.

\section{Compliance with ethical standards}

Conflict of interest The authors declare that they have no conflict of interest.

Open Access This article is licensed under a Creative Commons Attribution 4.0 International License, which permits use, sharing, adaptation, distribution and reproduction in any medium or format, as long as you give appropriate credit to the original author(s) and the source, provide a link to the Creative Commons license, and indicate if changes were made. The images or other third party material in this article are included in the article's Creative Commons license, unless indicated otherwise in a credit line to the material. If material is not included in the article's Creative Commons license and your intended use is not permitted by statutory regulation or exceeds the permitted use, you will need to obtain permission directly from the copyright holder. To view a copy of this license, visit http://creativecommons. org/licenses/by/4.0/

\section{References}

1. Snead MP, McNinch AM, Poulson AV, et al. Stickler syndrome, ocular-only variants and a key diagnostic role for the ophthalmologist. Eye. 2011;25:1389-1400.
2. Richards AJ, McNinch A, Martin H, et al. Stickler Syndrome and the vitreous phenotype: mutations in COL2A1 and COL11A1. Hum Mutat. 2010;31:E1461-71.

3. Acke FR, Dhooge IJ, Malfait F, De Leenheer EM. Hearing impairments in Stickler syndrome: a systematic review. Orphanet J Rare Dis. 2012;7:84.

4. Couchouron T, Masson C. Early-onset progressive osteoarthritis with hereditary progressive ophthalmopathy or Stickler syndrome. Jt Bone Spine. 2011;78:45-49.

5. Evans AK, Rahbar R, Rogers GF, Mulliken JB, Volk MS. Robin sequence: a retrospective review of 115 patients. Int J Pedatr Otorhinolaryngol. 2006;70:973-80.

6. Stickler GB, Hughes W, Houchin P. Clinical features of hereditary progressive arthro-ophthalmopathy (Stickler syndrome): a survey. Genet Med. 2001;3:192-6.

7. Richards AJ, Yates JR, Williams R, et al. A family with Stickler syndrome type 2 has a mutation in the COL11A1 gene resulting in the substitution of glycine 97 by valine in alpha 1 (XI) collagen. Hum Mol Genet. 1996;5:1339-43.

8. Sirko-Osadsa DA, Murray MA, Scott JA, Lavery MA, Warman ML, Robin NH. Stickler syndrome without eye involvement is caused by mutations in COL11A2, the gene encoding thealpha2 (XI) chain of type XI collagen. J Pediatr. 1998;132:368-71.

9. Richards AJ, Martin S, Yates JRW, et al. COL2A1 exon 2 mutations: relevance to the Stickler and Wagner syndromes. Br J Ophthalmol. 2000;84:364-71.

10. Van Camp G, Snoeckx RL, Hilgert N, et al. A new autosomal recessive form of Stickler syndrome is caused by a mutation in the COL9A1 gene. Am J Hum Genet. 2006;79:449-57.

11. Baker S, Booth C, Fillman C, et al. A loss of function mutation in the COL9A2 gene cause autosomal recessive Stickler syndrome. Am J Med Genet A. 2011;155:1668-72.

12. Faletra F, D'Adamo AP, Bruno I, et al. Autosomal recessive Stickler syndrome due to a loss of function mutation in the COL9A3 gene. Am J Med Genet A. 2014;164A:42-47.

13. Richards AJ, Fincham GS, McNinch A, et al. Alternative splicing modifies the effect of mutations in COL11A1 and results in recessive type 2 Stickler syndrome with profound hearing loss. J Med Genet. 2013;50:765-71.

14. Alzahrani F, Al Hazzaa SA, Tayeb H, Alkuraya FS. LOXL3, encoding lysyl oxidase-like 3 , is mutated in a family with autosomal recessive Stickler syndrome. Hum Genet. 2015;134:451-3.

15. Schrauwen I, Sommen M, Claes C, et al. Broadening the phenotype of LRP2 mutations: a new mutation in LRP2 causes a predominantly ocular phenotype suggestive of Stickler syndrome. Clin Genet. 2014;86:282-6.

16. Kingsley D. The TGF- $\beta$ superfamily: new members, new receptors, and new genetic tests of function in different organisms. Genes Dev. 1994;8:133-46.

17. Sasagawa S, Takabatake T, Takabatake Y, Muramatsu T, Takeshima K. Axes establishment during eye morphogenesis In Xenopus by coordinate and antagonistic actions of BMP4, Shh and RA. Genesis. 2002;33:86-96.

18. Huang J, Liu Y, Oltean A, Beebe D. BMP4 from the optic vesicle specifies murine retina formation. Dev Biol. 2015;402:119-26.

19. Bakrania P, Efthymiou M, Klein JC, et al. Mutations in BMP4 cause eye, brain, and digit developmental anomalies: overlap between the BMP4 and hedgehog signaling pathways. Am J Hum Genet. 2008;82:304-19.

20. Reis LM, Tyler RC, Schilter KF, et al. BMP4 loss-of-function mutations in developmental eye disorders including SHORT syndrome. Hum Genet. 2011;130:495-504.

21. Lumaka A, Van Hole C, Casteels I, et al. Variability in expression of a familial $2.79 \mathrm{Mb}$ microdeletion in chromosome 14q22.1-22.2. Am J Med Genet A. 2012;158A:1381-7. 
22. Hayashi S, Okamoto N, Makita Y, Hata A, Imoto I, Inazawa J. Heterozygous deletion at 14q22.1-q22.3 including the BMP4 gene in a patient with psychomotor retardation, congenital corneal opacity and feet polysyndactyly. Am J Med Genet A. 2008;146A:2905-10.

23. Nolen LD, Amor D, Haywood A, et al. Deletion at $14 \mathrm{q} 22-23$ indicates a contiguous gene syndrome comprising anophthalmia, pituitary hypoplasia, and ear anomalies. Am J Med Genet A. 2006; 140:1711-8.

24. Dunn NR, Winnier GE, Hargett LK, Schrick JJ, Fogo AB, Hogan BL. Haploinsufficient phenotypes in Bmp4 heterozygous null mice and modification by mutations in Gli3 and Alx4. Dev Biol. 1997;188:235-47.

25. Richards S, Aziz N, Bale S, et al. Standards and guidelines for the interpretation of sequence variants: a joint consensus recommendation of the American College of Medical Genetics and Genomics and the Association for Molecular Pathology. Genet Med. 2015;17:405-24.

26. Richards AJ, Scott JD, Snead MP. Molecular genetics of rhegmatogenous retinal detachment. Eye. 2000;16:388-92.

27. Bragdon B, Moseychuk O, Saldanha S, King D, Julian J, Nohe A. Bone morphogenetic proteins: a critical review. Cell Signal. 2011;23:609-20.

28. Neugebauer JM, Kwon S, Kim HS, et al. The prodomain of BMP4 is necessary and sufficient to generate stable BMP4/7 heterodimers with enhanced bioactivity in vivo. Proc Natl Acad Sci USA. 2015;112:E2307-2316.

29. Wyatt AAW, Osborne RJ, Stewart H, Ragge NK. Bone morphogenetic protein 7 (BMP7) mutations are associated with variable ocular, brain, ear, palate, and skeletal anomalies. Hum Mutat. 2010;31:781-7.

30. Takenouchi T, Nishina S, Kosaki R, et al. Concurrent deletions of BMP4 and OTX2 genes, two master genes in ophthalmogenesis. Eur J Med Genet. 2013;56:50-53.
31. Trousse F, Esteve P, Bovolenta P. BMP4 mediates apoptic cell death in the developing chick eye. J Neurosci. 2001;21:1292-301.

32. Behesti H, Holt JK, Sowden JC. The level of BMP4 signaling is critical for the regulation of distinct T-box gene expression domains and growth along the dorso-ventral axis of the optic cup. BMC Dev Biol. 2006;6:62.

33. Zhu Y, Oganesian A, Keene DR, Sandell LJ. Type IIA procollagen containing the cysteine-rich amino propeptide is deposited in the extracellular matrix of prechondrogenic tissue and binds to TGF- $\beta 1$ and BMP-2. J Cell Biol. 1999;144:1069-80.

34. Spickett C, Hysi P, Hammond CJ, et al. Deep intronic sequence variants in COL2A1 affect the alternative splicing efficiency of exon 2, and may confer a risk for rhegmatogenous retinal detachment. Hum Mutat. 2016;37:1085-96.

35. Goldman DC, Donley N, Christian JL. Genetic interaction between Bmp2 and Bmp4 reveals shared functions during multiple aspects of mouse organogenesis. Mech Dev. 2009; 126:117-27.

36. Paralkar VM, Vukicevic S, Reddi AH. Transforming growth factor beta type 1 binds to collagen IV of basement membrane matrix: implications for development. Dev Biol. 1991;143: 303-8.

37. Fincham GS, James S, Spickett C, et al. Posterior vitreous detachment and the posterior hyaloid membrane. Ophthalmology. 2018;125:227-36.

38. Neptune ER, Frischmeyer PA, Arking DE, et al. Dysregulation of TGF-beta activation contributes to pathogenesis in Marfan syndrome. Nat Genet. 2003;33:407-11.

39. Ramirez F, Rifkin D. Extracellular microfibrils; contextual platforms for TGF $\beta$ and BMP signalling. Curr Opin Cell Biol. 2009;21:616-22.

40. Sanna-Cherchi S, Westland R, Ghiggeri GM, Gharavi AG. Genetic basis of human congenital anomalies of the kidney and urinary tract. J Clin Invest. 2018;128:4-15. 